\title{
Technè
}

La science au service de l'histoire de l'art et de la préservation des biens culturels

41 | 2015

Arts textiles antiques et modernes. Approche scientifique et restauration

\section{Les costumes des élégants d'Antinoé conservés au musée des Tissus de Lyon : approche historique, analyses techniques et analyses de colorants}

Costumes worn by elegant society in Antinopolis now in the Musée des Tissus:

historical approach, analyses of techniques and dyes

Maximilien Durand, Marie-Hélène Guelton, Lore Troalen, Emily Pearson et Alison N. Hulme

\section{OpenEdition Journals}

Édition électronique

URL : http://journals.openedition.org/techne/4303

DOI : $10.4000 /$ techne.4303

ISSN : 2534-5168

Éditeur

C2RMF

Édition imprimée

Date de publication : 25 novembre 2015

Pagination : $32-45$

ISBN : 978-2-7118-6248-1

ISSN : $1254-7867$

Référence électronique

Maximilien Durand, Marie-Hélène Guelton, Lore Troalen, Emily Pearson et Alison N. Hulme, «Les costumes des élégants d'Antinoé conservés au musée des Tissus de Lyon : approche historique, analyses techniques et analyses de colorants », Technè [En ligne], 41 | 2015, mis en ligne le 08 août 2020, consulté le 11 mars 2021. URL : http://journals.openedition.org/techne/4303 ; DOI : https:// doi.org/10.4000/techne.4303

La revue Technè. La science au service de l'histoire de l'art et de la préservation des biens culturels est mise à disposition selon les termes de la Licence Creative Commons Attribution - Pas d'Utilisation Commerciale - Pas de Modification 4.0 International. 


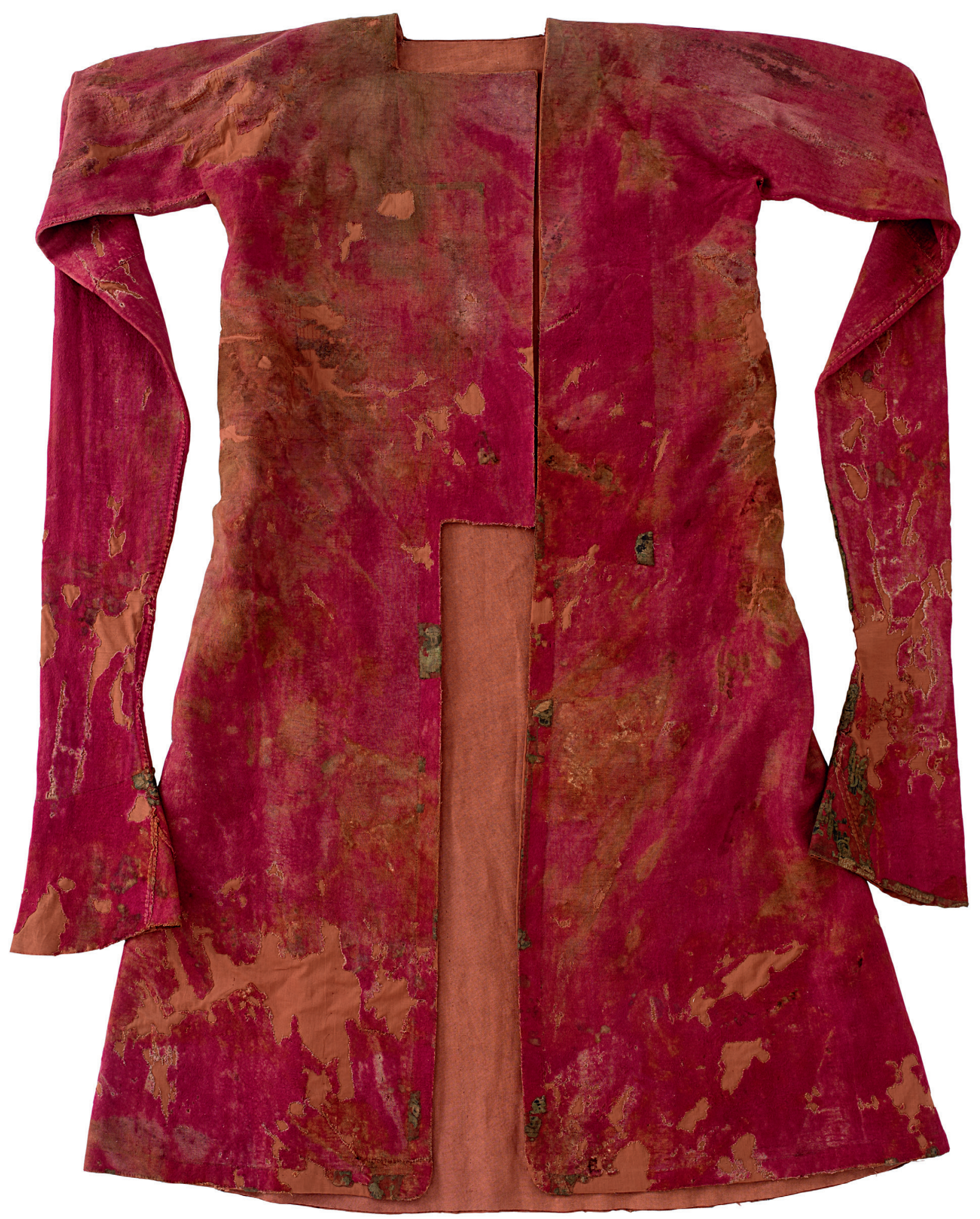

Fig. 1. Manteau du « haut dignitaire» (Achille). Antinoé, campagne de 1898, tombe B 281. Toile de laine grattée sur les deux faces et parements de soie (samit façonné et samit façonné «mi-soie »). Lyon, musée des Tissus, inv. MT 34872. @ Musée des Tissus/Pierre Verrier. 
Maximilien Durand

Marie-Hélène Guelton

Lore Troalen

Emily Pearson

Alison N. Hulme

\section{Les costumes des élégants d'Antinoé conservés au musée des Tissus de Lyon : approche historique, analyses techniques et analyses de colorants}

Costumes worn by elegant society in Antinopolis now in the Musée des Tissus: historical approach, analyses of techniques and dyes
Résumé. L'exposition Antinoé, à la vie, à la mode. Visions d'élégance dans les solitudes, organisée par le musée des Tissus en 2013, en partenariat avec le musée du Louvre, a permis de révéler la presque totalité du produit des fouilles de la campagne de 1898 sur le site d'Antinoé. Toutes les étoffes issues de cette campagne avaient été envoyées à Lyon, et oubliées dans les réserves du musée des Tissus. Pour la première fois, des costumes complets et des fragments de vêtements remarquables, provenant des quartiers de la nécropole où reposaient les fonctionnaires impériaux et la haute bourgeoisie de la cité antique, ont été présentés au public. Les costumes les plus exceptionnels, qui ont fait l'objet d'une analyse technique approfondie, ont également pu servir à caractériser les colorants employés à l'époque byzantine pour la production de luxe. Les analyses de colorants, effectuées par le Musée national d'Écosse et l'université d'Édimbourg, ont caractérisé notamment des rouges précieux (cochenille d'Arménie, kermès), des jaunes de flavonoïdes rares (bois de fustet) et des procédés tinctoriaux subtils (garance et indigo ou gaude, par exemple).

Mots-clés. Antinoé, colorants, analyse technique, coton de jeune pousse, soie tussah, torsion $Z$, toile à effet crêpe, toile grattée, Albert Gayet, Émile Guimet, byzantin, musée des Tissus.
Abstract. On display in the exhibition "Antinopolis: Beyond Life, Beyond Fashion - Shadows of Elegance in the Desert", organized by the Musée des Tissus, Lyon, in 2013, in partnership with the Louvre, were almost all of the articles uncovered in the excavations on the site of Antinopolis in 1898. All the fabrics found in these excavations were sent to Lyon, stored away in the Musée des Tissus, and forgotten. In this exhibition, complete costumes and fragments of remarkable garments, from the parts of the necropolis where imperial officials and members of the ancient city's uppermiddle class were buried, were shown to the public for the first time. The most exceptional costumes, which were subjected to an in-depth technical analysis, also enabled us to characterise the dyes used in the Byzantine period in the production of luxury fabrics. Notably, dye analysis carried out by National Museums Scotland and the University of Edinburgh showed the use of precious reds (Armenian cochineal, Kermes), rare flavonoid-rich yellows (young fustic) and subtle dye processes (using madder and indigo or weld, for example).

Keywords. Antinopolis, dyes, technical analysis, seed cotton, wild silk, Z-twist, crepe cotton, brushed cotton, Albert Gayet, Émile Guimet, Byzantine, Musée des Tissus.

\section{Le matériel conservé au musée des Tissus de Lyon et sa redécouverte}

En septembre 2013, le musée des Tissus de Lyon inaugurait l'exposition Antinoé, à la vie, à la mode. Visions d'élégance dans les solitudes ${ }^{1}$, dont le commissariat était assuré par Florence Calament, conservateur du patrimoine à la section copte du département des Antiquités égyptiennes du musée du Louvre, et Maximilien Durand, directeur du musée des Tissus et du musée des Arts décoratifs de Lyon. Elle a été organisée en partenariat avec le musée du Louvre ${ }^{2}$. Le projet de cette exposition faisait suite à la redécouverte d'un ensemble exceptionnel de costumes et de textiles exhumés dans la cité d'Antinoé, en Moyenne-Égypte, pour beaucoup inédits, conservés au musée des Tissus de Lyon.
Les premières explorations menées sur le site d'Antinoé en mars-avril 1896 par l'archéologue Albert Gayet (1856-1916), grâce à la volonté d'Émile Guimet (1836-1918) et aux subventions allouées par le musée qu'il avait fondé à Paris, s'étaient concentrées sur les ruines de la ville antique et le dégagement d'un temple ramesside ${ }^{3}$. Le matériel exposé au musée Guimet à l'issue de la première campagne - figurines en terres cuites, masques en plâtre, poteries -, ne laissait en rien deviner le véritable trésor que renfermait la cité. Durant la campagne suivante, entre février et avril 1897, Albert Gayet, bénéficiant d'une allocation doublée, poursuit ses travaux dans la zone urbaine où il découvre une chapelle d'Aménophis IV et deux temples d'époque romaine. Il commence surtout ses investigations vers le nord-est de la ville, en lisière des contreforts

Maximilien Durand, directeur du musée des Tissus et du musée des Arts décoratifs de Lyon (mdurand@museedestissus.com). Marie-Hélène Guelton, chargée des analyses techniques au musée des Tissus de Lyon, secrétaire générale technique du CIETA (guelton@mtmad.fr). Lore Troalen et Emily Pearson, Analytical Scientists, Collections Services Department, National Museums Scotland ; Edinburgh, UK (l.troalen@nms. ac.uk et ecpearson2@gmail.com).Alison N. Hulme, Senior Lecturer, EaStCHEM School of Chemistry ; Edinburgh, UK (Alison.Hulme@ed.ac.uk) 
de la chaîne arabique, et met au jour une nécropole, dans laquelle il identifie quatre quartiers, qu'il nomme A, B, C et D et qu'il croit correspondre aux périodes « égyptienne », « romaine », «byzantine » et « copte »; il extrait les premiers témoins de « costumes complets et souvent splendides ${ }^{4}$ », mais il est mal équipé et peu préparé à ce type de découverte si bien qu'il ne peut rapporter à Paris que des fragments. Aucun n'est d'ailleurs présenté dans l'exposition organisée au musée Guimet à l'occasion du onzième congrès international des Orientalistes qui se tient dans la capitale ${ }^{5}$. Émile Guimet a, en effet, d'autres projets pour ce matériel. Dès le 28 septembre 1897, l'amateur lyonnais sollicite la Chambre de Commerce de sa ville. Il fait don, pour le musée des Tissus, de « deux cadres contenant des étoffes trouvées à Antinoé (Égypte) et remontant à la période romaine ; quatre enveloppes contenant des fragments d'étoffes. Ces échantillons proviennent des fouilles récemment entreprises en Égypte par M. Gayet $(\ldots)^{6}$. » Émile Guimet espère convaincre la Chambre de Commerce de participer au financement de la campagne suivante, s'engageant «à réserver à la Chambre toutes les étoffes qui seront découvertes ultérieurement».

La Chambre de Commerce participe donc à l'entreprise sous la forme d'une souscription de deux mille francs, le musée Guimet finançant les mille autres francs qui permettent d'assurer la campagne. Albert Gayet, entre février et avril 1898, se concentre sur les quatre quartiers de la nécropole découverts l'année précédente. Les sépulcres maçonnés des quartiers $\mathrm{B}$ et $\mathrm{C}$ révèlent une population raffinée, comptant très certainement les fonctionnaires impériaux et les élégantes de la haute bourgeoisie antinoïte. À leur costume remarquable correspond du matériel funéraire soigné. Les étoffes extraites du quartier D, où les défunts sont inhumés en pleine terre, sont plus populaires. L'essentiel du matériel exhumé date du $\mathrm{IV}^{\mathrm{e}} \mathrm{au} \mathrm{VII}^{\mathrm{e}}$ siècle, soit la période byzantine, durant laquelle la cité a été particulièrement prospère. Albert Gayet s'attache à collecter le contenu entier de certaines sépultures, à l'exception des corps qui restent sur le site. Les cadavres sont dépouillés de leurs vêtements, et ce qui a survécu de l'opération de déshabillage est emporté à Paris. Avant même d'achever sa campagne, Albert Gayet priait Émile Guimet « de demander à la Chambre de Commerce qu'elle autorise une exposition de quinze jours ou trois semaines par exemple, lors de l'arrivée des caisses à Paris et du classement des objets rapportés. Elle ne pourrait que gagner à cette exposition temporaire à votre musée $^{7} »$. Albert Gayet était bien conscient du caractère inhabituel de ses découvertes, et de ce que révélait le contenu intègre de ces tombeaux anonymes de la civilisation qui les avait produits. «Avant d'en disperser les pièces, d'envoyer les étoffes à Lyon pendant que les souliers iront je ne sais où [...], il serait infiniment important de montrer au public artiste et lettré cet ensemble; d'étaler devant lui toutes les pièces d'un même costume, entourées de tous les objets retrouvés dans le même tombeau. »

La Chambre de Commerce accepte bien volontiers le principe d'une exposition parisienne qui se tient du 22 mai au 30 juin au musée Guimet. Le produit de la campagne est exposé dans dix-huit vitrines, situées à l'intérieur et sur le côté droit de la grande rotonde d'entrée du musée et au pied de l'escalier monumental menant à l'étage. Les fragments les plus remarquables, de soieries essentiellement, sont consultables dans un meuble à volets conçu tout spécialement pour cet usage. L'exposition donne lieu à la publication d'un catalogue sommaire, un petit fascicule non illustré de soixantequatre pages qui mentionne, après une brève introduction, le contenu de chacune des vitrines et du meuble à volets. C'est presque la seule publication de l'archéologue sur cette campagne qui s'avère être l'une des plus importantes pour la connaissance du site et de ses sépultures ${ }^{8}$.

Après l'exposition, les étoffes sont mises en caisses et, conformément à l'accord passé entre Émile Guimet et la Chambre de Commerce, expédiées à Lyon en 1899. Elles sont accompagnées par un courrier du 20 février 1899 signé de Léon de Milloué, proche collaborateur d'Émile Guimet et conservateur du musée Guimet, qui décrit la nature de l'envoi et la répartition dans les caisses : "Caisse 1 : les étoffes des vitrines $\mathrm{n}^{\circ} 1,2,4,5,6,7,8,9,12,14$ et 17 du catalogue ; 2 cartables renfermant 14 cartons avec soieries et étoffes collées. Caisse 2 : étoffes de la vitrine 18, objets non exposés et objets non catalogués $n^{\circ} 622,1207,1180,847,1913,974$ (?), 538, 581, 1310, 641, 644, 636 et 672. Caisse 3 : étoffes des vitrines 10 et 16,12 boîtes contenant des tours de cou, des coiffures et des serviettes frisées, 1 boîte rouge contenant le coussin romain $\mathrm{n}^{\circ} 112$; Objets non catalogués $\mathrm{n}^{\circ} 1297,1940$, 749,$758 ; 6$ pièces sans numéro ${ }^{9}$. »

Le contenu des caisses, à leur arrivée à Lyon, est partiellement identifié par le conservateur du musée des Tissus, Antonin Terme. Les vêtements les plus complets et les plus immédiatement identifiables sont extraits de leurs boîtes et exposés dans les galeries du musée, au second étage du Palais du Commerce. Quelques-unes sont décrites brièvement dans le premier guide des collections publié le $1^{\mathrm{er}}$ janvier 1902 par Raymond Cox ${ }^{10}$. La Chambre de Commerce participera au financement des campagnes ultérieures menées par Albert Gayet à Antinoé, par souscription à la Société française de fouilles archéologiques, créée le 14 janvier 1904, ou par l'attribution d'une somme de deux mille francs pour la campagne de 1908 « en retour de laquelle le directeur du musée historique des Tissus aurait le droit de choisir, le premier, les spécimens de tissus pouvant offrir de l'intérêt pour les collections du musée de la Chambre ${ }^{11}$ ». Par ailleurs, Émile Guimet sera particulièrement attentif à pourvoir le musée des Tissus des exemplaires d'étoffes les plus remarquables issues du site d'Antinoé, et l'institution bénéficie de sa générosité à plusieurs reprises en recevant des étoffes issues des campagnes de 1901, 1901-1902, 1903, 1906 et 1907.

L'exposition Antinoé, à la vie, à la mode. Visions d'élégance dans les solitudes a permis de redécouvrir une grande partie de ce matériel. Pour la première fois, le produit presque complet de la campagne de 1898 et les textiles provenant des campagnes suivantes ont pu être identifiés, inventoriés ou récolés, étudiés, analysés, restaurés et présentés au public. Chaque textile a d'abord été mis en relation avec sa description 
partielle dans les publications d'Albert Gayet. Pour cela, une étude systématique du vocabulaire parfois littéraire ou abscons de l'archéologue a dû être menée. Cela a permis de distinguer, parmi les fragments, ceux qui appartenaient aux costumes de défunts, et ceux qui relevaient de parures féminines. Puis chaque textile a été examiné en détail du point de vue de la qualité des matières employées, du soin apporté au filage, puis au tissage, aux finitions et à la confection pour les pièces de vêtement. Les analyses techniques détaillées, réalisées par Marie-Hélène Guelton selon la méthode du Centre international d'étude des textiles anciens, ont été publiées dans le catalogue de l'exposition.

\section{Parures des «fantômes d'Antinoé »: analyses techniques et analyses de colorants}

Albert Gayet était persuadé qu'on pouvait écrire une histoire de la cité d'Antinoé grâce aux costumes portés par ses défunts. Dans l'exposition qu'il organise en 1898, il s'efforce de classer ses trouvailles, vitrine par vitrine, pour livrer les grands chapitres de cette histoire, ponctués par les costumes complets de neuf adultes (tombes B 281, B 176, B 149, C 395, B 114, B 417, B 148, B 106 et C 350) et de deux enfants (tombes C 444 et $\mathrm{C} 412)^{12}$.

Dès les premiers temps de l'exposition estivale, Albert Gayet qualifie les morts dont il a rapporté les costumes complets à Paris. On voit ainsi apparaître, aux côtés de la « musicienne " (B 176), du «scribe » (C 395), du « haut fonctionnaire » (B 148) et des deux «amazones» (B 106 et C 350) qui sont déjà ainsi désignés dans le catalogue de l'exposition, un « haut dignitaire » (B 281), et des épithètes : la première « amazone » est qualifiée de « romaine» ou de «paienne », la seconde, de «chrétienne. » Rien, dans la sépulture du « haut dignitaire », n'indiquait la position qu'il avait occupée dans la ville, sinon son précieux manteau en laine grattée, rehaussé de parements de soie, et le luxe de son costume ; l'« amazone chrétienne » est ainsi appelée car sa robe comporte, sur les manches, des " dessins blancs et verts sur fond violet » où Albert Gayet reconnaît « des figures de saints guerriers".

Sans surprise, ce sont évidemment les costumes conférant aux défunts, par leur luxe ou leur caractère inhabituel, une individualité particulière, voire une véritable personnalité au point d'être revêtus de surnoms évocateurs par l'archéologue, qui ont révélé le plus grand intérêt archéologique. Pour compléter les analyses techniques effectuées au musée des Tissus, un partenariat a été établi avec le laboratoire scientifique du Musée national d'Écosse (National Museums Scotland) et le département de chimie de l'université d'Édimbourg (University of Edinburgh) pour identifier les colorants sur les vêtements qui semblaient les plus précieux, en concentrant les analyses sur les couleurs rouge, orangé et jaune observées sur les vêtements de la meilleure qualité. Ce sont eux qui semblaient devoir fournir les informations les plus intéressantes et les plus inédites.

\section{Analyses techniques}

\section{Les costumes d'hommes}

Parmi ces derniers se distinguent évidemment les manteaux d'hommes en laine cachemire, de couleur turquoise ou carmin, grattée après tissage, à longues manches et parements précieux. Le musée des Tissus conserve six manteaux de ce type, plus ou moins complets, quatre de couleur carmin, provenant des sépultures B $281^{13}$ (fig. 1), C $395^{14}$, B $114^{15}$ (fig. 2) et B $148^{16}$, et deux turquoise, provenant des tombes B $139^{17}$ (fig. 3 ) et B $288^{18}$. Ils sont taillés dans une toile en laine cachemire de torsion $\mathrm{Z}$, en chaîne comme en trame. La toile obtenue a une forte densité de trame qui recouvre presqu'entièrement la chaîne. Le tissu est ensuite gratté à l'aide de chardons sur les deux faces. Parmi les manteaux de couleur carmin, celui du « haut fonctionnaire » de la tombe B 148 se singularise par sa structure en sergé de 2 lie 2, direction Z. La diagonale en $\mathrm{Z}$ du sergé est accentuée par la torsion $\mathrm{Z}$ des fils et la trame double. Les manteaux de couleur turquoise, eux, ont tous été tissés avec une trame double, de faible torsion. Le tassage dans la foule des fils doubles avait pour but de faciliter le grattage à la tombée du métier, tout en formant une étoffe épaisse et souple. Le grattage est effectué par un léger mouvement de droite à gauche (direction $\mathrm{Z}$ ), puis de gauche à droite, dans le sens chaîne (longitudinal),

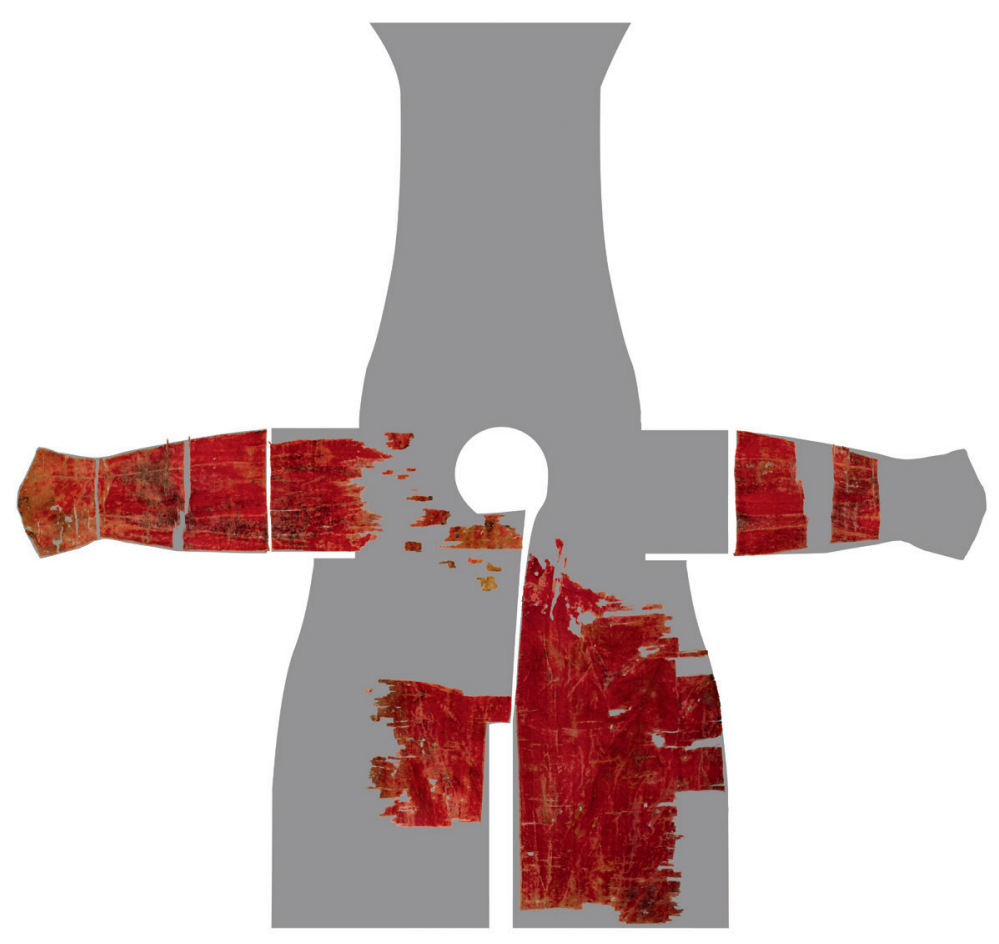

Fig. 2. Fragments de manteau d'homme. Antinoé, campagne de 1898, tombe B 114. Toile de laine grattée sur les deux faces et parements de soie (samit façonné). Lyon, musée des Tissus, inv. MT 2013.0.17 et MT 2014.0.23. (C) Musée des Tissus/Pierre Verrier. 


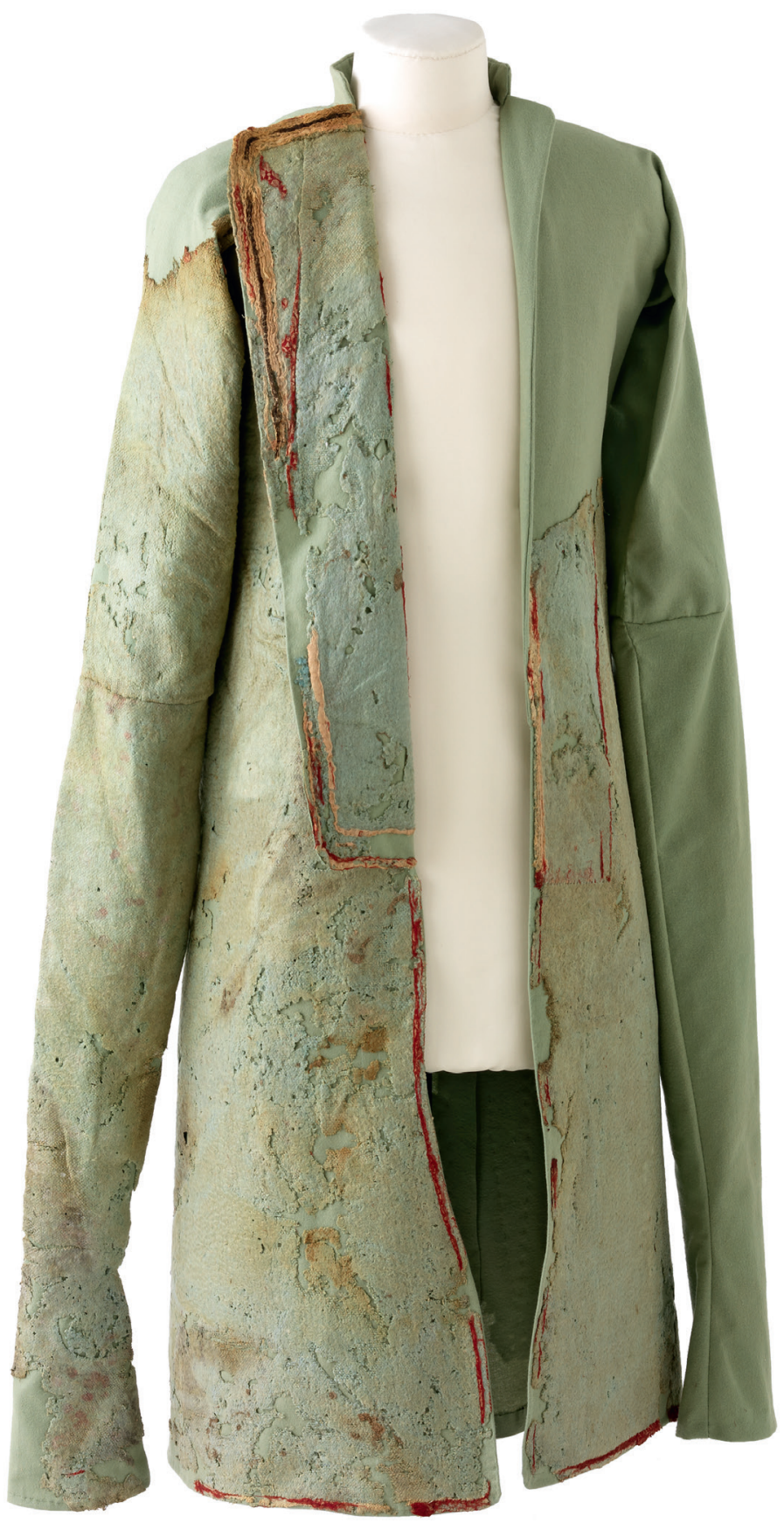

Fig. 3. Manteau d'homme. Antinoé, campagne de 1898, tombe B 139. Toile de laine grattée sur les deux faces et parements de cordelettes de lin, de fine toile de laine et de samits façonnés " mi-soie ». Lyon, musée des Tissus, inv. MT 2013.0.6. (C) Musée des Tissus/Pierre Verrier.

la trame dominante étant peignée en formant des ondulations qui accentuent la brillance de la fibre, la profondeur et les nuances du coloris. Le tissu duveteux évoque alors l'aspect d'une fourrure (fig. 1). Les étoffes utilisées pour la confection des manteaux de couleur carmin ont été teintes en pièce, comme on peut l'observer quand on extrait un fil de chaîne

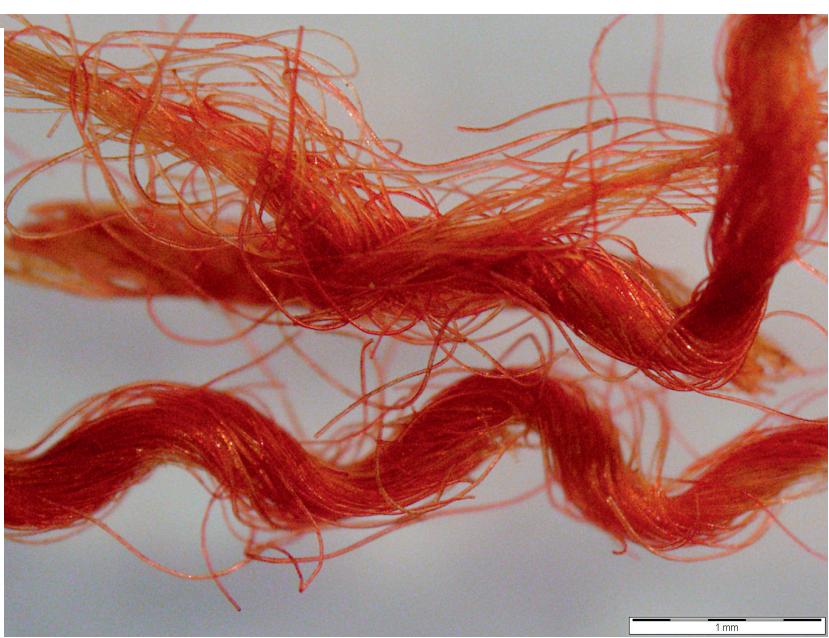

Fig. 4. Fibres extraites du manteau issu de la tombe B 148 (Lyon, musée des Tissus, inv. MT 2013.0.33). Grossissement 20 x. ( NMS/ Lore Troalen.

du tissage, moins bien teint que la trame (fig. 4), alors que les manteaux de couleur turquoise sont teints en fil, comme en témoignent, lorsque les extrémités de la laize sont conservées, les passées de trame non teinte. Une fois la laize tombée du métier et grattée, le tailleur procédait au plan de découpe, puis à l'assemblage du manteau, et appliquait les parements. Ceux-ci sont constitués de soieries pour les exemplaires carmin taillés dans les toiles les plus fines, ou d'applications de cordelettes de lin, de toiles fines, de samit de soie ou mi-soie, ou de galons de laine pour les exemplaires en toile plus épaisse.

Les analyses de colorants, pour les costumes d'hommes, ont porté sur les toiles carmin des manteaux B 114, B 148 et d'un manteau non identifié ${ }^{19}$, sur les parements en toile de laine fine des manteaux B 148, carmin, B 139, turquoise, et sur un fragment de manteau non identifié, également turquoise ${ }^{20}$.

\section{Les costumes de femmes}

Le vestiaire féminin révélé par la campagne de 1898 semble lui aussi avoir été très précieux. Pour la première fois ont pu être étudiés et analysés des costumes de femmes réalisés dans la même toile cachemire grattée après tissage, de couleur carmin, que les costumes d'hommes. Il s'agit de la robe de la défunte issue de la tombe C $408^{21}$ et du manteau de celle inhumée dans la tombe B $417^{22}$. Les analyses de colorants ont également concerné les costumes des défuntes issues des tombes C $339^{23}$, C $370^{24}$ et B $106^{25}$ (l'« Amazone païenne »), parce qu'ils présentaient une qualité de tissage ou de finition supérieure, ainsi que C $408^{26}$ et B $200^{27}$, parce que leur couleur rose orangé est peu fréquente dans les textiles égyptiens d'époque byzantine. Par ailleurs, ces deux derniers offrent une contexture raffinée : le premier, issu de la tombe C 408, est en laine de forte torsion $\mathrm{Z}$ pour réaliser une toile crêpe, 
très souple, barrée de lin aux extrémités (fig. 5). Sa couleur accompagne avec subtilité celle de la robe carmin issue de la même tombe. Le second, provenant de la tombe B 200, est aussi tissé en fine toile crêpe mais avec une laine de torsion $\mathrm{S}$, barrée aux extrémités de laine non teinte plus serrée, ce qui ajoute un tombé différent au tissu. Sa bordure se caractérise par une tresse transversale formée par les fils de chaîne qui se terminent en franges câblées.

Enfin, des analyses ont également été réalisées sur des textiles provenant de sépultures plus populaires, sur une toile de laine à franges insérées et décor de tapisserie extraite de la tombe D $1261^{28}$, d'une typologie inhabituelle, et sur une robe de femme, plutôt commune, extraite durant la sixième campagne de $1901^{29}$

Par le choix de ces vêtements, tous inédits au moment de l'étude, nous souhaitions vérifier si la qualité des fils et du tissage était en corrélation avec l'identification de colorants plus ou moins précieux, comme nous le supposions.

\section{Analyse des colorants par chromatographie liquide (PDA-UPLC)}

La caractérisation des colorants naturels dans les textiles archéologiques se fonde sur l'identification de composés marqueurs permettant de différencier diverses espèces végétales ou animales ${ }^{30}$. Cependant, plusieurs sources colorantes présentent des constituants majeurs similaires et l'identification d'une espèce précise ne pourra donc se faire que par celle de composés mineurs associés. Ainsi le flavone lutéoline (Lut) et son glucoside (Lut-7-O-glu) sont présents dans de nombreux jaunes de flavonoïdes ${ }^{31}$. Pour cette raison, seules les présences additionnelles du flavone apigénine (Api) et du flavone $O$-méthylé chrysoériol (Cry) dans les extraits après hydrolyse acide permettent d'identifier avec certitude le jaune de gaude (Reseda luteola L.) ${ }^{32}$. Enfin, la petite taille des échantillons prélevés pour l'analyse combinée à la sensibilité de certains colorants aux processus de photo-dégradation et de photo-oxydation $^{33}$, qui sont particulièrement critiques pour les jaunes de flavonoïdes, peut souvent limiter l'identification de la source végétale utilisée pour le bain de teinture.

Un ensemble de quarante-sept prélèvements de fibres textiles teintes a été effectué sur une sélection de quinze textiles provenant de vêtements d'hommes et de femmes (Tableaux I et II). Les échantillons ont été préparés en suivant un protocole d'extraction ne préservant pas les glucosides ${ }^{34}$, tandis que pour certains échantillons, il a été possible de compléter cette analyse par un protocole d'extraction préservant les glucosides ${ }^{35}$. Les extraits ont ensuite été analysés par Chromatographie Liquide à Ultra Performance (UPLC ${ }^{\circledR}$ ) avec détection en UV-Vis (210-500 nm), une technique d'analyse particulièrement adaptée pour l'étude d'échantillons dégradés, car elle permet d'obtenir des limites de détections de l'ordre du $\mathrm{ng}^{36}$. Les conditions expérimentales ont été optimisées pour l'analyse de colorants jaunes à base de flavonoïdes et ont été récemment publiées ${ }^{37}$.

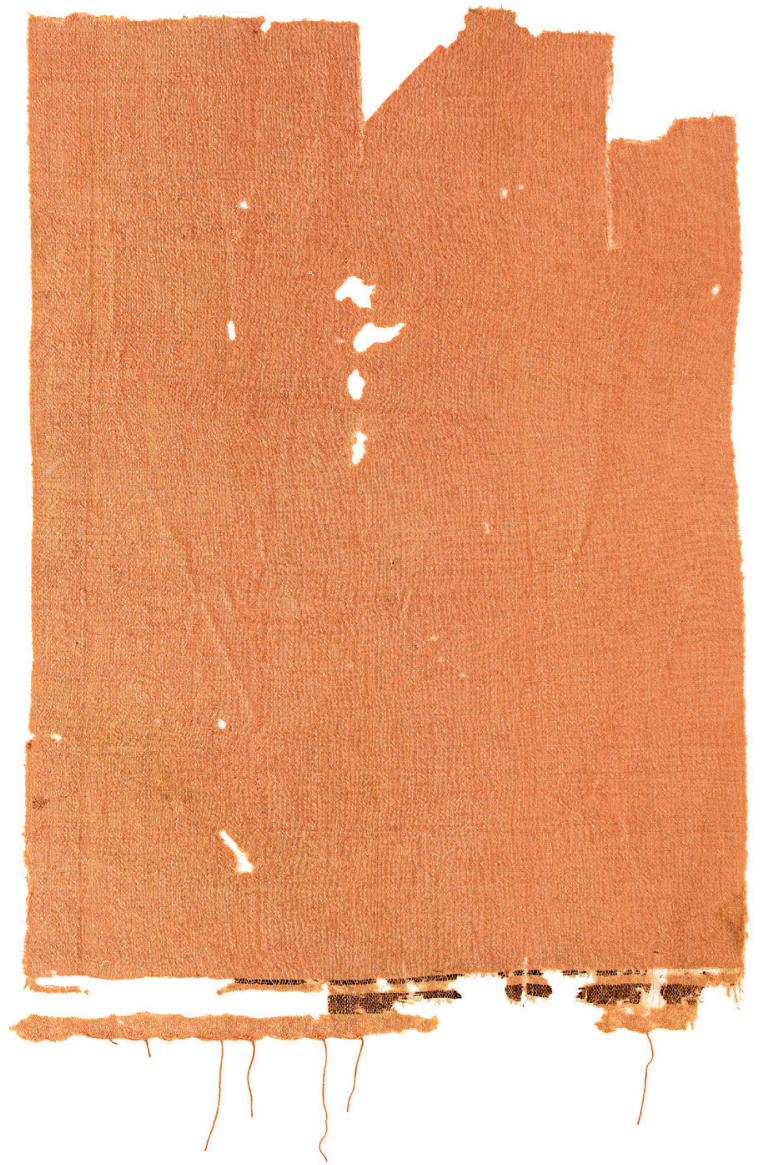

Fig. 5. Manteau de femme. Antinoé, campagne de 1898, tombe C 408 Toile de laine à effet crêpe, barrée de lin. Lyon, musée des Tissus, inv. MT 2013.0.15. (C) Musée des Tissus/Pierre Verrier.

\section{Les colorations jaunes}

Plusieurs sources de jaunes de flavonoïdes ont été caractérisées. Bien que la gaude (Reseda luteola L.) soit présente dans la majorité des échantillons jaunes, orange et verts, une autre source de flavonoïdes a été mise en évidence : le bois de fustet (Cotinus Coggygria Scop.).

La majorité des colorations à base de flavonoïdes identifiées dans les textiles de femmes sont moins dégradées que celles des costumes d'hommes. Ainsi, il a été possible d'établir avec certitude l'usage du jaune de gaude dans plusieurs textiles de femmes, grâce à la présence dans les extraits hydrolysés des flavones lutéoline (Lut) et apigénine (Api), ainsi que du flavone $O$-méthylé chrysoériol $(\mathrm{Cry})^{38}$. Les proportions relatives d'apigénine et de chrysoériol varient entre $8-15 \%$ et 2-8\% respectivement, selon le degré de photo-dégradation du colorant sur la fibre ${ }^{39}$. Enfin, le bois de fustet (Cotinus Coggygria Scop.), aussi appelé bois de sumac, a été caractérisé dans la bordure ocre du manteau d'homme MT 2013.0.33 où il est associé à de la garance. La présence de bois de fustet est particulièrement intéressante, car cette espèce n'est pas native en Égypte, mais est répandue en Méditerranée ${ }^{40}$. Elle se caractérise par l'association dans l'extrait hydrolysé du flavonol fisétine (Fis) et de l'aurone sulfurétine ${ }^{41}$ (Sul). 


\section{Les colorations rouges}

Les colorations rouges ont été obtenues par l'utilisation de sources végétales et animales. Dans la majorité des cas, la garance a été utilisée pour obtenir les couleurs orangées, parfois associée à des jaunes de flavonoïdes ou bien avec un sur-teint d'indigo pour les colorations pourpres. La garance des teinturiers a été caractérisée par la présence dans les extraits hydrolysés d'alizarine (Ali) et de purpurine (Pur). Les quantités d'anthraquinones sont connues pour être très variables dans les racines de garance ${ }^{42}$. Ainsi, dans six échantillons analysés, une prédominance du colorant purpurine après hydrolyse acide a été observée. Pour deux de ces échantillons, la quantité d'alizarine n'a été observée que dans un ratio $1: 9$ avec la purpurine (MT 47555.1 et MT 47555.2). Cette faible quantité d'alizarine pourrait indiquer soit l'utilisation de garance sauvage (Rubia peregrina), qui est pratiquement dépourvue d'alizarine ${ }^{43}$, soit l'utilisation de garance plus âgée ${ }^{44}$. Enfin, dans le cas des échantillons analysés par une méthode d'extraction plus douce, il n'a pas été possible de caractériser de précurseurs glucidiques. Des résultats similaires ont été rapportés dans de récentes analyses sur des textiles d'Antinoé conservés au musée du Louvre, pour lesquels l'absence de précurseurs glucidiques avait été associée à une préparation des textiles dans un bain de teinture ayant permis leur hydrolyse enzymatique par fermentation ${ }^{45}$.

Enfin, dans les tissus les plus prestigieux, deux sources colorantes d'origine animale ont été caractérisées pour la réalisation des teintes rouges vives : la cochenille, probablement d'Arménie (Porphyrophora hamelii Brandt), et le kermès

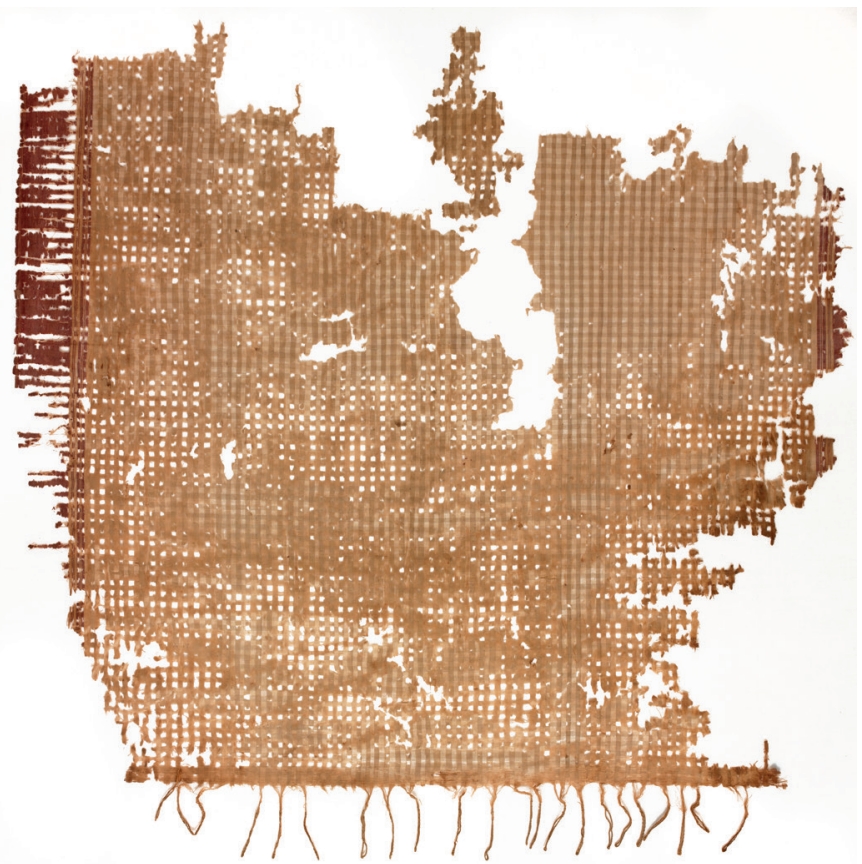

Fig. 6. Écharpe de femme. Antinoé, campagne de 1901-1902. Toile rayée et quadrillée en coton et soie sauvage. Lyon, musée des Tissus, inv. MT 2013.0.64. (C) Musée des Tissus/Pierre Verrier.
(Kermes vermilio Planchon), parfois associés à de la garance selon les tonalités. Ces deux sources ont été mises en évidence par la présence d'acide carminique (Car) dans la cochenille d'Arménie et l'association des acides kermésique (Ker) et flavokermésique (Flk) dans le kermès ${ }^{46}$.

\section{Les colorations vertes et bleues}

Les colorations vertes analysées ont été obtenues par des mélanges de bleus et de jaunes. Dans la majorité des cas, il s'agit de sur-teints d'indigo sur jaune de gaude (Reseda Luteola L.), ou bien d'indigo seul pour les teintes bleues foncées. La présence d'indigo a été confirmée par la détection d'indigotine (Ind) dans les extraits après hydrolyse acide.

\section{Conclusion}

La campagne d'analyse de colorants, ciblée sur les vêtements les plus prestigieux issus des fouilles de 1898, a confirmé la grande maîtrise des procédés tinctoriaux des artisans égyptiens de l'époque byzantine. Elle montre surtout la corrélation évidente qui existe entre la qualité des fibres utilisées, comme le duvet de chèvre cachemire ou la laine présentant un filage particulièrement soigné (dans le cas des toiles à effet crêpe, par exemple, pour les manteaux de femmes), et le choix de colorants précieux ou rares, cochenille d'Arménie, kermès ou bois de fustet, ce dernier ayant été identifié ici pour la première fois sur une étoffe égyptienne, ainsi que de nuances subtiles, obtenues avec des colorants plus usuels (manteaux rose orangé issus des tombes C 408 et B 200). Elle révèle aussi des procédés homogènes, comme la préparation du fil, le tissage ou la confection, sur des vêtements dont on avait longtemps pensé qu'ils avaient été importés parce que la forme du vêtement ou les matières premières le constituant n'étaient pas indigènes. Les ateliers de luxe importaient certainement le duvet cachemire, certaines matières tinctoriales, d'Orient (cochenille, kermès) ou de Méditerranée occidentale (bois de fustet), mais réalisaient le tissage, les finitions et la confection dans la ville pour répondre aux exigences d'une population raffinée. Le montage des métiers utilisés pour le tissage, les procédés (lisières, contexture des étoffes, finitions des bordures) et les caractéristiques de la confection (rabats de couture, fils employés pour les assemblages, points de couture), présentent une grande homogénéité par rapport à l'ensemble du matériel exhumé des nécropoles de la ville, autorisant à reconnaître une production locale dans ces vêtements de luxe. Les fouilles d'Antinoé ont, par ailleurs, révélé quelques rares exemples de vêtements importés, comme une exceptionnelle écharpe de femme venue d'Inde ${ }^{47}$ (fig. 6), en toile rayée et quadrillée tissée en soie tussah et coton de jeune pousse ${ }^{48}$, que leurs caractéristiques techniques distinguent immédiatement du corpus appréhendé en partenariat avec le laboratoire scientifique du Musée national d'Écosse et l'université d'Édimbourg. 


\section{Analyse technique et caractérisation des colorants}

\section{Tableau I. Costumes d'hommes}

Synthèse des sources végétales et animales identifiées dans les costumes d'hommes. Les chromophores correspondent aux colorants identifiés dans les extraits après hydrolyse acide et reconstitution pour l'analyse par PDA-UPLC.

(Les composés sont annotés de la façon suivante : Ind : indigotine; Lut : lutéoline ; Api : apigénine ; Cry : chrysoériol ; Ali : alizarine ; Pur : purpurine ; Fis : fisétine ; Sul : sulfurétine ; Car : acide carminique; Flk : acide flavokermésique ; Ker : acide kermésique.)

\begin{tabular}{|c|c|c|c|}
\hline Analyse technique & $\begin{array}{l}\text { Prélèvement : fibre, } \\
\text { couleur }\end{array}$ & $\begin{array}{l}\text { Chromophores identifiés } \\
\text { (PDA-UPLC)* }\end{array}$ & Colorants \\
\hline \multicolumn{4}{|c|}{$\begin{array}{l}\text { Fragment de manche de manteau d'homme } \\
\qquad \begin{array}{c}\text { MT } 2013.0 .34 \\
\left(\text { p. } 203^{49}\right)\end{array}\end{array}$} \\
\hline \multirow{4}{*}{$\begin{array}{l}\text { Toile dominante trame, grattée } 2 \text { faces. } \\
\text { Chaîne: laine } \mathrm{Z} \text { turquoise } ; 7 / \mathrm{cm} \text {. Trame: } \\
\text { laine } \mathrm{Z} \text { turquoise, fil double } ; 6-7 / \mathrm{cm} \text {. } \\
\text { Bordure : toile fine dominante trame, } \\
\text { grattée } 2 \text { faces. } \\
\text { Chaîne: laine rouge } ; 18 / \mathrm{cm} \text {. } \\
\text { Trame: laine rouge } ; 64 / \mathrm{cm} \text {. }\end{array}$} & $\begin{array}{l}\text { Laine turquoise } \\
\text { Châ̂ne }\end{array}$ & Traces de Lut et Ind & $\begin{array}{l}\text { Sur-teint d'indigo sur jaune } \\
\text { de flavonoïdes } \\
\text { (source non identifiée, gaude?) }\end{array}$ \\
\hline & $\begin{array}{l}\text { Laine turquoise } \\
\text { Trame }\end{array}$ & Traces de Lut et Ind & $\begin{array}{l}\text { Sur-teint d'indigo sur jaune } \\
\text { de flavonoïdes } \\
\text { (source non identifiée, gaude?) }\end{array}$ \\
\hline & Laine rouge Chaîne & $\begin{array}{l}\text { Car, dcIV et dcVII et un } \\
\text { colorant rouge non identifié } \\
\left(\mathrm{R}_{\mathrm{t}}=18.85 \mathrm{~min},\left[\lambda_{\max }=229,\right.\right. \\
261(\mathrm{~s}), 310,411(\mathrm{~s}), \\
489 \mathrm{~nm}]) .\end{array}$ & $\begin{array}{l}\text { Cochenille, probablement cochenille } \\
\text { d'Arménie ; le colorant rouge } \\
\text { non identifié pourrait correspondre } \\
\text { à une contamination }\end{array}$ \\
\hline & Laine rouge Trame & Traces de Car & $\begin{array}{l}\text { Cochenille, probablement cochenille } \\
\text { d'Arménie }\end{array}$ \\
\hline \multicolumn{4}{|c|}{$\begin{array}{l}\text { Fragment de samit mi-soie appliqué sur le manteau d'homme } \\
\qquad \text { B } 139 \\
\text { MT } 2013.0 .6\end{array}$} \\
\hline $\begin{array}{l}\text { Samit façonné } 2 \text { lats, liage sergé } 2 \text { lie } \\
1 \mathrm{Z} \text {. } \\
\text { Chaînes : } 1 \mathrm{P}, 1 \mathrm{~L} ; \mathrm{P} \text { et } \mathrm{L} \text {, soie } \mathrm{Z} \\
\text { jaunâtre ; découpure : } 1 \mathrm{P} ; 26 \mathrm{P} \text { et L/ } \\
\text { cm. } \\
\text { Trames : } 1,2 ; 1,2 ; 1: \text { laine } \mathrm{Z} \text { rouge ; } \\
2 \text { : soie STA } 2 \text { bouts crème ; découpure : } \\
1 \text { passée ; } 48-51 \text { passées/cm. }\end{array}$ & $\begin{array}{l}\text { Laine rouge Trame, } \\
\text { samit mi-soie }\end{array}$ & Car, dcIV et dcVII & $\begin{array}{l}\text { Cochenille, probablement cochenille } \\
\text { d'Arménie }\end{array}$ \\
\hline \multicolumn{4}{|c|}{$\begin{array}{l}\text { Fragment de manteau d'homme } \\
\text { Tombe non identifiée } \\
\text { SN } \\
\text { (non publié) }\end{array}$} \\
\hline \multirow{2}{*}{$\begin{array}{l}\text { Toile dominante trame, grattée } 2 \text { faces. } \\
\text { Chaîne : laine } \mathrm{Z} \text { rouge rosé } ; 12-14 / \mathrm{cm} \text {. } \\
\text { Trame: laine } \mathrm{Z} \text { rouge } ; 32-34 / \mathrm{cm} \text {. }\end{array}$} & Laine orange Chaîne & Traces de Car & $\begin{array}{l}\text { Fibre très photo-dégradée pour } \\
\text { permettre une identification - } \\
\text { possible cochenille d'Arménie? }\end{array}$ \\
\hline & Laine orange Trame & $\begin{array}{l}\text { Car } \\
\text { Traces de Ali, Pur }\end{array}$ & $\begin{array}{l}\text { Cochenille, probablement cochenille } \\
\text { d'Arménie avec résidu de garance }\end{array}$ \\
\hline
\end{tabular}




\begin{tabular}{|c|c|c|c|}
\hline \multicolumn{4}{|c|}{$\begin{array}{l}\text { Revers droit du manteau de « haut fonctionnaire » } \\
\text { B } 148 \\
\text { MT } 2013.0 .33 \\
\text { (p. } 200)\end{array}$} \\
\hline $\begin{array}{l}\text { Sergé } 2 \text { lie } 2, \mathrm{Z} \text { endroit, gratté } 2 \text { faces. } \\
\text { Chaîne : laine fort } \mathrm{Z} \text {, rouge orangé ; } \\
20-22 / \mathrm{cm} \text {. } \\
\text { Trame : laine } \mathrm{Z} \text {, rouge, double ; } 9-10 / \mathrm{cm} \text {. } \\
\text { Teint pièce (?) }\end{array}$ & $\begin{array}{l}\text { Laine rouge } \\
\text { Trame, sergé }\end{array}$ & $\begin{array}{l}\text { Car } \\
\text { Ali, Pur }(65: 35)\end{array}$ & $\begin{array}{l}\text { Garance, avec présence résiduelle } \\
\text { de cochenille, probablement } \\
\text { d'Arménie }\end{array}$ \\
\hline \multirow{5}{*}{ 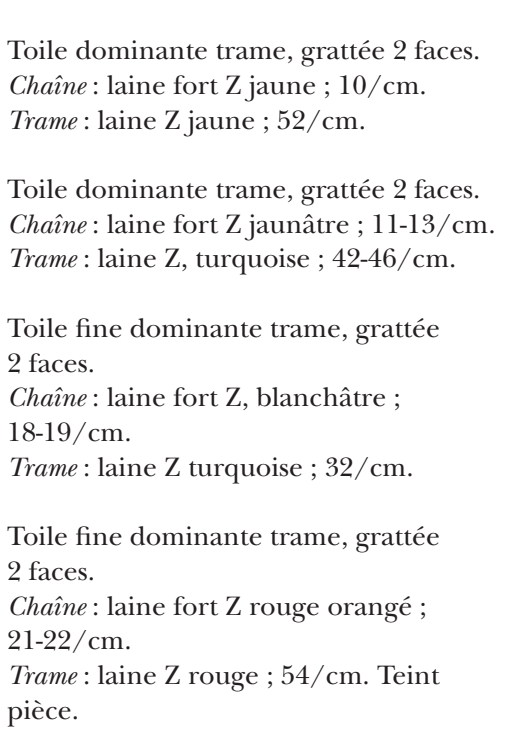 } & $\begin{array}{l}\text { Laine jaune Bordure, } \\
\text { bande ocre }\end{array}$ & $\begin{array}{l}\text { Ali, Pur }(82: 18) \\
\text { Fis, Sul } \\
\text { deux flavonols non } \\
\text { identifiés : } 1\left(\mathrm{R}_{\mathrm{t}}=10,63 \mathrm{~min},\right. \\
{\left[\lambda_{\max }=237,308(\mathrm{~s}), 343\right.} \\
\mathrm{nm}]) ; 2\left(\mathrm{R}_{\mathrm{t}}=11,37 \mathrm{~min},\right. \\
{\left[\lambda_{\max }=244,308(\mathrm{~s}), 350\right.} \\
\mathrm{nm}])\end{array}$ & Garance et bois de fustet \\
\hline & $\begin{array}{l}\text { Laine blanche Chaîne } \\
\text { de bordure turquoise }\end{array}$ & Traces de Lut & $\begin{array}{l}\text { Jaune de flavonoïdes } \\
\text { (source non identifiée, gaude?) }\end{array}$ \\
\hline & $\begin{array}{l}\text { Laine turquoise } \\
\text { Trame }\end{array}$ & Ind & $\begin{array}{l}\text { Coloration à base d'indigo, pas de } \\
\text { traces de colorants jaunes détectées }\end{array}$ \\
\hline & $\begin{array}{l}\text { Laine rouge Toile } \\
\text { grattée }\end{array}$ & $\begin{array}{l}\text { Car } \\
\text { Ali, Pur (35:65) }\end{array}$ & $\begin{array}{l}\text { Cochenille, probablement cochenille } \\
\text { d'Arménie et garance }\end{array}$ \\
\hline & $\begin{array}{l}\text { Laine rouge Trame, } \\
\text { toile grattée }\end{array}$ & $\begin{array}{l}\text { Car } \\
\text { Traces de Ali }\end{array}$ & $\begin{array}{l}\text { Cochenille, probablement cochenille } \\
\text { d'Arménie et résidu de garance }\end{array}$ \\
\hline \multicolumn{4}{|c|}{$\begin{array}{l}\text { Manteau d'homme } \\
\text { B } 114 \\
\text { MT 2013.0.17 } \\
\text { (p. 168) }\end{array}$} \\
\hline \multirow{3}{*}{$\begin{array}{l}\text { Toile dominante trame, grattée } 2 \text { faces. } \\
\text { Chaîne: laine fort } \mathrm{Z} \text { beige rosé ; } 7-8 / \mathrm{cm} \text {. } \\
\text { Trames : laine } \mathrm{Z} \text { rouge ; } 30-32 / \mathrm{cm} \text {. }\end{array}$} & $\begin{array}{l}\text { Laine beige rosé } \\
\text { Chaîne }\end{array}$ & $\begin{array}{l}\text { Ali et Pur }(90: 10) \\
\text { Traces de Flk, Ker }\end{array}$ & Garance et résidu de kermès \\
\hline & $\begin{array}{l}\text { Laine rouge Laine } \\
\text { grattée }\end{array}$ & $\begin{array}{l}\text { Flk, Ker } \\
\text { Traces de Ali et Pur }\end{array}$ & Kermès et résidu de garance \\
\hline & Laine rouge Trame & Traces de Flk, Ker, Ali, Pur & $\begin{array}{l}\text { Possible kermès avec résidu } \\
\text { de garance }\end{array}$ \\
\hline
\end{tabular}

* Les valeurs indiquées entre parenthèses correspondent aux quantités relatives extraites à 350 nm pour les jaunes de flavonoïdes (gaude) et à $254 \mathrm{~nm}$ pour les colorants à base d'anthraquinones (garance). 


\section{Tableau II. Costumes de femmes}

Synthèse des sources végétales et animales identifiées dans les costumes de femmes. Les chromophores correspondent aux colorants identifiés dans les extraits après hydrolyse acide et reconstitution pour l'analyse par PDA-UPLC.

(Les composés sont annotés de la façon suivante : Ind : indigotine ; Lut : lutéoline ; Api : apigénine ; Cry : chrysoériol ; Ali : alizarine ; Pur : purpurine ; Fis : fisétine; Sul : sulfurétine ; Car : acide carminique ; Fkl : acide flavokermésique ; Ker : acide kermésique.)

\begin{tabular}{|c|c|c|c|}
\hline Analyse technique & $\begin{array}{l}\text { Prélèvements: fibre, } \\
\text { couleur, description }\end{array}$ & $\begin{array}{l}\text { Chromophores identifiés } \\
\text { (PDA-UPLC) }^{*}\end{array}$ & Colorants \\
\hline \multicolumn{4}{|c|}{$\begin{array}{l}\text { Fragments de manteau de femme } \\
\text { C } 339 \\
\text { MT } 47555.1 \text { et MT } 47555.2 \\
\text { (p. 152) }\end{array}$} \\
\hline \multirow{2}{*}{$\begin{array}{l}\text { Toile crêpe barrée. } \\
\text { Chaîne : laine crêpe } \mathrm{S} ; 15-17 / \mathrm{cm} \text {. } \\
\text { Trame barrée : laine crêpe } \mathrm{S} \text {; laine } \mathrm{S} \text { blanche ; } \\
\text { 10-24/cm, } 32 \text { (barrures). Barrures sur } 9 \mathrm{~cm} \text { aux } \\
\text { extrémités. Lisières : } 2 \text { sur } 0,6 \mathrm{~cm} ; 3 \text { cordelines } \\
\text { de } 9,7,9 \text { fils. Finitions : chaîne tressée } \\
\text { par } 3 \text { groupes de } 7-8 \text { fils ; franges retors Z } \\
\text { de 2-8 fils, ou câblé S } 2 \text { retors Z de } 8 \text { fils. }\end{array}$} & Laine rouge Chaîne & Ali, Pur (8:92) & Garance** \\
\hline & Laine rouge Trame & Ali, Pur (10:90) & Garance** \\
\hline \multicolumn{4}{|c|}{$\begin{array}{l}\text { Manteau de femme et bourrelet } \\
\text { C } 370 \\
\text { MT 49189; MT 51398.28; MT 2013.0.16 } \\
\text { (p. 162) }\end{array}$} \\
\hline \multirow{4}{*}{$\begin{array}{l}\text { Toile crêpe et tapisserie incluse (relais, duites } \\
\text { courbes, ressauts, trame enroulée, perfilage). } \\
\text { Chaîne : laine crêpe S jaune ; } 11-12 / \mathrm{cm} \text {. } \\
\text { Trames : continues, discontinues ; laine crêpe } \mathrm{S} \\
\text { jaune ; laine } \mathrm{S} \text { blanc (barrure) ; laine } \mathrm{S}, 2 \text { bleus, } \\
\text { rouge, orange, jaune, vert, marron ; lin S non } \\
\text { teint ; } 13-14 \text { (fond), } 44 / \mathrm{cm} \text { (tapisserie). } \\
\text { Lisières : } 2 \text { sur } 0,7 \mathrm{~cm}: 3 \text { cordelines de } 5-6 \text { fils. } \\
\text { Tresse à franges bouclées : rapportées le long } \\
\text { d'une lisière tresse rouge/jaune et franges } \\
\text { bouclées de } 2 \mathrm{~cm} \text {, laine S rouge. } \\
\text { Bourrelet rapporté : tressage à plat } 21 \text { brins } \\
\text { de } 3 \text { bouts en sorte de sergé ; laine gros retors S } \\
\text { de } 3 \mathrm{~S} \text {, rouge. }\end{array}$} & Laine jaune Chaîne & Lut, Api, Cry (91.4: 6: 2.6) & Jaune de gaude \\
\hline & Laine jaune Trame & Lut, Api, Cry (87: 8: 5) & Jaune de gaude \\
\hline & $\begin{array}{l}\text { Laine rouge Frange } \\
\text { bouclée }\end{array}$ & $\begin{array}{l}\text { Lut, Api, Cry (59: 30: 10) } \\
\text { Ali, Pur (29: 71) }\end{array}$ & $\begin{array}{l}\text { Garance** et jaune } \\
\text { de gaude }\end{array}$ \\
\hline & $\begin{array}{l}\text { Laine rouge } \\
\text { Fil bourrelet }\end{array}$ & $\begin{array}{l}\text { Ali, Pur (42: } 58) \\
\text { Traces Lut }\end{array}$ & $\begin{array}{l}\text { Garance et jaune } \\
\text { de flavonoïdes (source } \\
\text { non identifiée, gaude?) }\end{array}$ \\
\hline \multicolumn{4}{|c|}{$\begin{array}{c}\text { Manteau de l'« Amazone païenne » B } 106 \\
\text { MT 2013.0.35 } \\
\text { (p. 206) }\end{array}$} \\
\hline \multirow{3}{*}{$\begin{array}{l}\text { Toile dominante trame et tapisserie incluse } \\
\text { (toile à relais, duites courbes, ressauts, } \\
\text { perfilages). } \\
\text { Chaîne: laine fort } S \text { non teint ; } 6-7 / \mathrm{cm} \text {. } \\
\text { Trames : continues, discontinues ; laine } S \text { non } \\
\text { teint (fond) ; laine } S \text { lie-de-vin, blanchâtre ; } \\
22-30 \text { (fond), } 39-40 / \mathrm{cm} \text { (tapisserie). } \\
\text { Lisières : } 2 \text { sur } 0,8 \mathrm{~cm} ; 3 \text { cordelines de } 2 \text { fils. } \\
\text { Finitions : franges et gros cordons de structure } \\
\text { complexe. }\end{array}$} & $\begin{array}{l}\text { Laine lie-de-vin } \\
\text { Trame }\end{array}$ & $\begin{array}{l}\text { Ali, Pur (25:75) } \\
\text { Ind }\end{array}$ & $\begin{array}{l}\text { Sur-teint d'indigo } \\
\text { sur garance }{ }^{* *}\end{array}$ \\
\hline & $\begin{array}{l}\text { Laine fond toile } \\
\text { Chaîne }\end{array}$ & Traces Pur & Probablement non teint \\
\hline & $\begin{array}{l}\text { Laine fond toile } \\
\text { Trame }\end{array}$ & Pas de colorants détectés & Probablement non teint \\
\hline
\end{tabular}




\begin{tabular}{|c|c|c|c|}
\hline \multicolumn{4}{|c|}{$\begin{array}{l}\text { Toile de laine à décor de franges insérées et tapisserie } \\
\text { D 1261 } \\
\text { MT 2013.0.38 } \\
\text { (p. 226) }\end{array}$} \\
\hline \multirow{2}{*}{$\begin{array}{l}\text { Toile dominante trame à franges insérées } \\
\text { et tapisserie incluse (toile barrée à relais, } \\
\text { duites courbes, perfilages, ressauts). } \\
\text { Chaîne : laine } \mathrm{S} \text { jaune ; } 8-9 / \mathrm{cm} \text {. } \\
\text { Trames continues, discontinues ; laine } \mathrm{S} \text { jaune ; } \\
\text { laine } \mathrm{S} \text { lie-de-vin, blanc, jaune ; } 34 \text { (fond), } \\
52 / \mathrm{cm} \text { (tapisserie). } \\
\text { Lisière: } 1 \text { sur } 0,7 \mathrm{~cm} ; 3 \text { cordelines de } 2 \text { fils. } \\
\text { Franges insérées au tissage tous les } 36-38 \text { coups } \\
\text { de toile : laine } S \text { jaune. Franges rouges } \\
\text { aux extrémités : laine } S \text { rouge. } \\
\text { Finitions : gros cordon câblé } S \text { de } 3 \text { cordons Z } \\
\text { de } 16 \text { fils de chaîne ( } 4 \text { groupes de } 4 \text { ). }\end{array}$} & $\begin{array}{l}\text { Laine lie-de-vin } \\
\text { Trame }\end{array}$ & $\begin{array}{l}\text { Traces Lut } \\
\text { Ali, Pur (40:60) } \\
\text { Ind }\end{array}$ & $\begin{array}{l}\text { Sur-teint d'indigo } \\
\text { sur garance** et jaune } \\
\text { de flavonoïdes (source } \\
\text { non identifiée, gaude?) }\end{array}$ \\
\hline & $\begin{array}{l}\text { Laine jaune } \\
\text { Frange }\end{array}$ & $\begin{array}{l}\text { Lut } \\
\text { Traces de Ali et Pur }\end{array}$ & $\begin{array}{l}\text { Traces de jaune } \\
\text { de flavonoïdes (source } \\
\text { non identifiée, gaude?) } \\
\text { et de garance }\end{array}$ \\
\hline \multicolumn{4}{|c|}{$\begin{array}{l}\text { Robe de femme } \\
\text { DUL } 1382 \\
\text { (p. } 344)\end{array}$} \\
\hline \multirow{3}{*}{$\begin{array}{l}\text { Toile barrée, barrée discontinue et tapisserie } \\
\text { incluse (louisine de } 2 \text { fils à relais, duites } \\
\text { courbes, trame enroulée, ressauts). } \\
\text { Chaîne } \text { lin } \mathrm{S} \text { non teint ; } 18 / \mathrm{cm} \text {. Trames } \\
\text { continues, discontinues ; lin } \mathrm{S} \text { brunâtre non } \\
\text { teint (fond) ; laine } \mathrm{S} \text { rouge, vert, bleu, jaune, } \\
\text { ocre, violet ; lin S non teint ; } 16 \text { (fond), } \\
48-56 / \mathrm{cm} \text { (tapisserie). Lisières : } 2 \text {; fil simple. }\end{array}$} & Laine rouge Trame & Ali, Pur (34:66) & Garance** \\
\hline & Laine verte Trame & $\begin{array}{l}\text { Lut, Api, Cry } \\
\text { Ind } \\
\text { Traces de Ali, Pur }\end{array}$ & $\begin{array}{l}\text { Sur-teint d'indigo sur jaune } \\
\text { de gaude et résidu } \\
\text { de garance }\end{array}$ \\
\hline & Laine bleue Trame & Pas de colorants détectés & Probablement indigo \\
\hline \multicolumn{4}{|c|}{$\begin{array}{c}\text { Fragment de manteau de femme } \\
\text { C } 408 \\
\text { MT } 2013.0 .15 \\
\text { (p. 160) }\end{array}$} \\
\hline \multirow{3}{*}{$\begin{array}{l}\text { Toile crêpe barrée. } \\
\text { Chaîne: laine fort } \mathrm{Z} \text { rose saumon ; } 21 / \mathrm{cm} \text {. } \\
\text { Trame: laine } \mathrm{Z} \text { rose saumon ; } 16 / \mathrm{cm} ; \text { lin } \\
\mathrm{S} \text { brun foncé fil triple ; barrures sur } 3 \mathrm{~cm} \text {. } \\
\text { Finitions : franges retors } \mathrm{Z} \text { de } 3 \text { groupes } \mathrm{S} \\
\text { de } 7-8 \text { fils Z. }\end{array}$} & $\begin{array}{l}\text { Laine rose saumon } \\
\text { Chaîne }\end{array}$ & Ali, Pur (76:24) & Garance \\
\hline & $\begin{array}{l}\text { Laine rose saumon } \\
\text { Trame }\end{array}$ & Ali, Pur (74:26) & Garance \\
\hline & $\begin{array}{l}\text { Lin brun } \\
\text { Trame }\end{array}$ & Traces de Ali & Traces de garance \\
\hline \multicolumn{4}{|c|}{$\begin{array}{l}\text { Fragment de manteau de femme } \\
\text { B } 200 \\
\text { MT 2013.0.50 } \\
\text { (p. 292) }\end{array}$} \\
\hline \multirow{2}{*}{$\begin{array}{l}\text { Toile crêpe barrée (toile et louisine de } 2 \text { fils). } \\
\text { Chaîne: laine crêpe } \mathrm{S} \text { orange } ; 11 / \mathrm{cm} \text {. Trame } \\
\text { barrée : laine crêpe } \mathrm{S} \text { orange } ; 15 / \mathrm{cm} ; \text { laine } \\
\mathrm{S} \text { non teint } ; 35 / \mathrm{cm} ; \text { barrures sur } 10,7 \mathrm{~cm} \text {. } \\
\text { Lisière: } 1 \text { sur } 0,5 \mathrm{~cm} ; 3 \text { cordelines de } 4 \text { fils. } \\
\text { Finitions : tresse transversale par } 3 \text { groupes de } \\
\text { chaîne et franges câblées } \mathrm{S} \text { de } 2 \text { retors } \mathrm{S} \text { de } 3 \mathrm{~S} \text {. }\end{array}$} & Laine orange Chaîne & $\begin{array}{l}\text { Lut, traces de Api } \\
\text { Ali, Pur }(73: 27)\end{array}$ & $\begin{array}{l}\text { Garance et jaune de } \\
\text { flavonoïdes (source } \\
\text { non identifiée, gaude ?) }\end{array}$ \\
\hline & Laine orange Trame & $\begin{array}{l}\text { Lut } \\
\text { Ali, Pur (72:28) }\end{array}$ & $\begin{array}{l}\text { Garance et jaune de } \\
\text { flavonoïdes (source } \\
\text { non identifiée, gaude ?) }\end{array}$ \\
\hline
\end{tabular}




\begin{tabular}{|c|c|c|c|}
\hline \multicolumn{4}{|c|}{$\begin{array}{l}\text { Trois fragments d'une robe } \\
\text { C } 408 \\
\text { MT 2013.0.14; MT } 28520.49 \\
\text { (p. 156) }\end{array}$} \\
\hline \multirow{3}{*}{$\begin{array}{l}\text { Robe : toile dominante trame, grattée } 2 \text { faces. } \\
\text { Chaîne: laine fort } \mathrm{Z} \text { beige rosé ; } 13 / \mathrm{cm} \text {. } \\
\text { Trame: laine } \mathrm{Z} \text { rouge foncé ; } 40 / \mathrm{cm} \text {. } \\
\text { Carré : Tapisserie, toile dominante trame à } \\
\text { relais, duites courbes, ressauts, trame enroulée. } \\
\text { Chaîne: lin } \mathrm{S} \text {, non teint ; } 11-12 / \mathrm{cm} \text {. } \\
\text { Trame discontinue : lin } \mathrm{S} \text { non teint simple } \\
\text { et double ; laine } \mathrm{Z} \text { marron ; } 56-58 / \mathrm{cm} \text {. } \\
\text { Lisières : } 2 ; 2 \text { fils de lin doubles. } \\
\text { Couture: lin retors } \mathrm{Z} 2 \mathrm{~S} \text { non teint. }\end{array}$} & $\begin{array}{l}\text { Laine rouge Chaîne, } \\
\text { grattée }\end{array}$ & Flk, Ker & Kermès \\
\hline & $\begin{array}{l}\text { Laine orange } \\
\text { Trame }\end{array}$ & Traces Flk & Possibles traces de kermès \\
\hline & Laine brune Trame & Pas de colorants détectés & Source non identifiée \\
\hline \multicolumn{4}{|c|}{$\begin{array}{c}\text { Robe de femme } \\
\text { B } 417 \\
\text { MT } 2013.0 .21 \\
\text { (p. 174) }\end{array}$} \\
\hline \multirow{6}{*}{$\begin{array}{l}\text { Robe : toile crêpe barrée et tapisserie incluse. } \\
\text { Chaîne: laine crêpe } \mathrm{S} \text { jaune ; } 16 / \mathrm{cm} \text {. } \\
\text { Trames continue, discontinues : laine } \mathrm{S} \text { jaune } \\
\text { (fond) ; laine } \mathrm{S} \text { bleu foncé, brun ; lin } \mathrm{S} \\
\text { non teint ; } 21-22 \text { (fond) ; } 56 / \mathrm{cm} \text { (tapisserie } \\
\text { incluse) ; barrures sur } 0,5 \mathrm{~cm} \text { (épaules). } \\
\text { Lisière: } 1 \text { sur } 0,7 \mathrm{~cm} ; 4 \text { cordelines : } 3 \text { à } 5 \text { fils, } \\
1 \text { à } 2 \text { fils. } \\
\text { Galon appliqué : tapisserie, toile dominante } \\
\text { trame à relais, duites courbes, ressauts, } \\
\text { perfilages ( ?). } \\
\text { Chaîne: laine } S \text { jaune clair ; } 12 / \text { cm. } \\
\text { Trames discontinues : laine bleu, rouge, jaune, } \\
\text { vert ; } 32 \text { / cm. Lisières : } 2 \text {; } 2 \text { cordelines de } 2 \text { fils. }\end{array}$} & Laine jaune Chaîne & Lut, Api, Cry (88: 7: 4) & Jaune de gaude \\
\hline & Laine jaune Trame & $\begin{array}{l}\text { Lut, Api, Cry (88: 9: 3) } \\
\text { Ali }\end{array}$ & $\begin{array}{l}\text { Jaune de gaude avec résidu } \\
\text { de garance }\end{array}$ \\
\hline & $\begin{array}{l}\text { Laine bleue Trame, } \\
\text { galon bleu foncé }\end{array}$ & Ind & Indigo \\
\hline & $\begin{array}{l}\text { Laine jaune Chaîne, } \\
\text { galon }\end{array}$ & Lut, Api, Cry (77: 15: 8) & Jaune de gaude \\
\hline & $\begin{array}{l}\text { Laine jaune Trame, } \\
\text { galon }\end{array}$ & $\begin{array}{l}\text { Lut, Api, Cry, (89: 8: 2) } \\
\text { Traces Ali }\end{array}$ & Jaune de gaude \\
\hline & Laine verte Trame, galon & $\begin{array}{l}\text { Lut, Api, Cry (93: 5: 2) } \\
\text { Ind }\end{array}$ & $\begin{array}{l}\text { Sur-teint d'indigo sur jaune } \\
\text { de gaude }\end{array}$ \\
\hline & $\begin{array}{c}\text { Manteau de fen } \\
\text { B } 417 \\
\text { MT 2013.0.2 } \\
\text { (p. 176) }\end{array}$ & & \\
\hline $\begin{array}{l}\text { Toile dominante trame, grattée } 2 \text { faces. Galon } \\
\text { rapporté. } \\
\text { Chaîne } \text { : laine fort } \mathrm{Z} \text {, rouge-orange ; } 18-19 / \mathrm{cm} \text {. } \\
\text { Trame } \text { laine } Z \text { roure } \cdot 48 / \mathrm{cm} \text { iòre. }\end{array}$ & $\begin{array}{l}\text { Laine rouge-orange } \\
\text { Trame }\end{array}$ & $\begin{array}{l}\text { Flk, Ker (impure). } \\
\text { Traces de colorants rouges } \\
\text { non identifiés }\end{array}$ & Possible kermès \\
\hline $\begin{array}{l}1 \text { sur } 0,6 \mathrm{~cm}, 4 \text { cordelines de } 9 \text { fils puis } 6-8 \text {. } \\
\text { Teint en pièce ( ?). } \\
\text { Finitions: franges câblées } S \text { de } 2 \text { câblés } Z \\
\text { de } 2 \text { retors de } 3 \text { bouts } Z \text {. } \\
\text { Galon : toile dominante chaîne, rayée. Franges } \\
\text { rapportées le long d'une lisière. }\end{array}$ & $\begin{array}{l}\text { Laine rouge } \\
\text { Franges }\end{array}$ & $\begin{array}{l}\text { Co-élution de Flk and Ker } \\
\text { (impure). } \\
\text { Traces de colorants rouges } \\
\text { non identifiés }\end{array}$ & Possible kermès \\
\hline
\end{tabular}

\footnotetext{
* Les valeurs indiquées entre parenthèses correspondent aux quantités relatives extraites à 350 nm pour les jaunes de flavonoïdes (gaude) et à 254 nm pour les colorants à base d'anthraquinones (garance).

** Correspond aux échantillons de garance où le colorant purpurine est présent majoritairement dans les extraits après hydrolyse acide.
} 


\section{Notes}

1. L'exposition bénéficiait du label « Exposition d'intérêt national » délivré par le ministère de la Culture et de la Communication.

2. Exp. Lyon, 2013

3. Sur le site d'Antinoé et sa redécouverte par Albert Gayet, on consultera Calament, 2005, et Calament, 2013.

4. Courrier à Émile Guimet, en date du 15 avril 1897, rédigé depuis l'Hôtel Roya du Caire (Archives du musée Guimet) ; cité dans Calament, 2013, p. 26.

5. Gayet, 1897-1 ; Gayet, 1897-2 ; Gayet, 1897-3 ; Gayet, 1897-4.

6. Courrier du 30 septembre 1897 ; archives de la Chambre de Commerce et d'Industrie de Lyon.

7. Courrier à Émile Guimet, en date du 3 mars 1898 (Archives du musée Guimet) ; cité dans Calament, 2013, p. 29.

8. Gayet, 1898-1 ; pour les autres publications de l'archéologue sur cette campagne, voir Gayet, 1898-2 et Gayet, 1898-3.

9. Archives de la Chambre de Commerce et d'Industrie de Lyon.

10. Cox, 1902.

11. Archives de la Chambre de Commerce et d'Ind ustrie de Lyon.

12. Sur l'organisation de l'exposition de 1898 par Albert Gayet, voir Durand, 2013.

13. Inv. MT 34872 ; Exp. Lyon, 2013, n 20 , p. 98-103.

14. Inv. MT 34872 bis; ibid., $\mathrm{n}^{\circ} 34$, p. 136-141.

15. Inv. MT 2013.0.17 et MT 2014.0.23 ; ibid., $\mathrm{n}^{\circ} 47$, p. 168-171; depuis la fin de l'exposition, d'autres fragments importants de ce manteau ont été redécouverts. Ils avaient été déposés à Stockholm quelques décennies auparavant, et y demeuraient toujours. En 1965, en effet, Agnès Geijer, qui s'intéressait aux vêtements en laine grattée découverts à Antinoé, consulta les nombreux fragments du musée des Tissus et convainquit l'institution de les déposer, pour étude et restauration, à l'atelier du Riksantikvarieämbetet och Statens historiska museet de Stockholm. L’opération s'est faite sans inventaire préalable des fragments ni identification des vestiges. Les manteaux du « haut dignitaire " Achille (tombe B 281 ; inv. MT 34872) et du "scribe " Pamias (tombe C 395 ; inv. MT 34872 bis) purent être isolés de ce lot. Ils firent l'objet d'une remarquable restauration. Le premier revint au musée des Tissus en 1968, le second resta en dépôt à Stockholm, en dédommagement de la restauration, jusqu'en 1993. Lors de la restauration, en 1989, du manteau de couleur turquoise de l'occupant de la tombe B 288 (inv. MT 47554), d'autres fragments turquoise et carmin furent restitués par le musée de Stockholm au musée des Tissus. Parmi eux se trouvait le grand revers droit du manteau du « haut fonctionnaire » de la tombe B 148 (inv. MT 2013.0.33), identifiable grâce à ses parements appliqués et aux restes de la soierie qui le garnissait, identique aux fragments qui étaient conservés séparément du vêtement (inv. MT
40315). Le manteau complet de ce « haut fonctionnaire " (inv. MT 2014.0.22), celui du défunt de la tombe B 114 (MT 2014.0.23), des fragments du manteau de la tombe $\mathrm{C}$ 322 (inv. MT 2014.0.24) et la jambière droite du «scribe " Pamias (inv. MT 2014.0.21) ont naguère été redécouverts à Stockholm, où ils étaient restés, sans qu'aucun acte de dépôt ne signale leur présence. Ils avaient donc totalement échappé à l'attention des chercheurs. Ils ont heureusement pu rejoindre les collections du musée des Tissus en 2014, qui est ainsi rentré en possession de l'ensemble des vestiges antinoïtes déposés à Stockholm.

16. Inv. MT 2013.0.33 et MT 2014.0.22 ; ibid., $\mathrm{n}^{\circ} 64$, p. 200-203. 127

17. Inv. MT 2013.0.6 ; ibid., n ${ }^{\circ} 29$, p. 120 167.

18. Inv. MT 47554 ; ibid., n 46 , p. 164 -

19. Inv. SN

20. Inv. MT 2013.0.34; ibid., p. 203.

21. Inv. MT 2013.0.14 et MT 18520.49;

ibid., $\mathrm{n}^{\circ} 42$, p. 156-159.

22. Inv. MT 2013.0.22; ibid., $\mathrm{n}^{\circ}$ 51,

p. 176-178.

23. Inv. MT 475545.1 et MT 475545.2 ;

ibid., $\mathrm{n}^{\circ} 39$, p. 152-154.

24. Inv. MT 49189, MT 51398.28 et MT

2013.0.16 ; ibid., $\mathrm{n}^{\circ} 45$, p. 162-163.

25. Inv. MT 2013.0.35; ibid., $\mathrm{n}^{\circ} 66$, p. 206-208.

26. Inv. MT 2013.0.15; ibid., $\mathrm{n}^{\circ} 43$, p. 160-161.

27. Inv. MT 2013.0.50; ibid., $\mathrm{n}^{\circ} 111$, p. 292-293.

28. Inv. MT 2013.0.38; ibid., $\mathrm{n}^{\circ} 78$, p. 226-228. 346.

29. Inv. DUL 1382 ; ibid., n ${ }^{\circ} 142$, p. 344-

30. Ferreira et al. (2004).

31. Cardon (2003), p. 146-154 ; Ferreira (2002), p. 82-116.

32. Peggie et al. (2008).

33. Ferreira (2002), p. 117-181 ; Hulme et al. (2003, in press)

34. Protocole adapté de Wouters \& Verhecken (1989a), avec une solution d'acide hydrochlorique concentrée ( $\mathrm{HCl}: \mathrm{H}_{2} \mathrm{O}: \mathrm{MeOH}$ ), 2:1:1, volume/volume, voir Troalen et al. (2014) pour le protocole détaillé.

35. Wouters, Grzywacz, Claro (2011), protocole combinant une extraction avec une solution d'acide oxalique et une extraction avec une solution d'acide hydrochlorique concentrée (voir note 34 ).

36 . Troalen (2013), p. 61-106; Troalen et al. (2014), op. cit. (note 34).

37. Ibid., pour les conditions

expérimentales détaillées.

38. Peggie et al. (2008), op. cit. (note 32).

39. Peggie (2006), p. 163-201; Troalen

(2013), op. cit. (note 36), p. 113-150.

40. Cardon (2003), p. 161-164.

41. Peggie (2006), p. 51-109.

42. Sanyova (2000).

43. Wouters (2001).

44. Sanyova (2000) ; Sanyova (2001), p. 103-178.

45. Nowik 2013, p. 134-137.

46. Wouters \& Verhecken (1989a), op. cit. (note 34); Wouters \& Verhecken (1989b).
47. Campagne de 1901-1902; inv. MT 2013.0.64

48. Analyses réalisées par Dominique de Reyer, au Laboratoire de recherche des Monuments historiques.

49. Les numéros de pages renvoient au catalogue de l'exposition où sont publiés les textiles avec leurs notices complètes : Exp. Lyon, 2013.

\section{Références bibliographiques}

Exp. Lyon, 2013, Antinoé, à la vie, à la mode. Visions d'élégance dans les solitudes [Exposition. Lyon, musée des Tissus, 2013], Calament F. et Durand M. (dir.).

Calament F., 2005, La révélation d'Antinoé par Albert Gayet. Histoire, archéologie, muséographie, Bibliothèque d'études coptes, 18, 2 vol., IFAO, Le Caire.

Calament F., 2013, « La résurrection d'un coin d'Égypte ignoré ", dans Exp. Lyon, 2013, p. 24-34.

Cardon D., 2003, Le monde des teintures naturelles, Belin, Paris.

Cox R., Catalogue sommaire des collections du musée historique des Tissus dressé sous la direction de M. Antonin Terme, directeur, par Raymond Cox, attaché au musée, chargé du cours de décoration des étoffes, Rey, Lyon, 1902.

Durand M., 2013, « Introduction aux notices du catalogue de l'exposition Antinoé, à la vie, à la mode. Visions d'élégance dans les solitudes ", dans Exp. Lyon, 2013, p. 36-42.

Ferreira E. S. B., McNab H., Hulme A. N. et Quye A., 2004, "The Natural Constituent of Historical Textile Dyes", Chemical Society Reviews, 33, p. 329-336.

Gayet A., 1897-1, «Correspondance. Les fouilles archéologiques en Égypte, en 1896-1897 ", Revue de l'Art ancien et moderne, 1/1, p. 79.

Gayet A., 1897-2, « Correspondance. Les fouilles du musée Guimet ", Revue de l'Art ancien et moderne, 1/2, p. 188-189.

Gayet A., 1897-3, «Compte-rendu des fouilles effectuées à Antinoé au cours de l'hiver 1896-1897 ", Annales du musée Guimet, 26/3, p. 55-58.

Gayet A., 1897-4, «Catalogue des principaux objets recueillis au cours des fouilles et entrés dans les collections du musée ", Annales du musée Guimet, 26/3, p. 59-62, pl. XIX-XXV.

Gayet A., 1898-1, Ministère de l'Instruction publique et des Beaux-Arts. Musée Guimet. Catalogue des objets recueillis à Antinoé pendant les fouilles de 1898 et exposés au musée Guimet du 22 mai au 30 juin 1898, Ernest Leroux, Paris.

Gayet A., 1898-2, « Le trésor de la nécropole d'Antinoé à l'exposition du musée Guimet ", Revue des Arts décoratifs, 18, p. 225-231.

Gayet A., 1898-3, «Un tour en Égypte », À travers le monde, 49-50, p. 385-388 et 393-396.

Hulme A. N., McNab H., Peggie D. et Quye A., 2003, "The Chemical Characterisation of Aged and Un-aged 
Fibre Samples Dyed with Sawwort (Serratula tinctoria) Using PDA-HPLC and HPLC ESI MS", Dyes in History and Archaeology, 22 (à paraître en $2015 / 2016$ ).

Peggie D. A., Hulme A. N., McNab H. et Quye A., 2008, "Towards the identification of characteristic minor components from textiles dyed with weld (Reseda luteola L.) and those dyed with Mexican cochineal (Dactylopius coccus Costa)", Microchimica Acta, 162, p. 371-380.

Nowik W., 2013, "Cheveux et couvre-chefs textiles : caractérisation des colorants », dans Antinoé. Momies, textiles, céramiques et autres Antiques. Musée du Louvre éditions, Paris, p. 134-137.

Sanyova, J., 2000, «Etude des pigments organiques préparés à partir des racines des rubiacées européennes ", dans Proceedings of Art et chimie, la couleur, J. Goupy, J.-P. Mohen (dir.), CNRS, Paris, p. 14-17.

Troalen L. G., Peggie D. A., Phillips A. S., Barran P. E. et Hulme A. N., 2014,

"Historical Textile Dyeing with Genista tinctoria L.: a Comprehensive Study by UPLC and MS/MS Analysis", Analytical Methods, 6, p. 8915-8923. Wouters J. et Verhecken A., 1989-1, "The Coccid Insect Dyes: HPLC and Computerized Diode-array Analysis of Dyed Yarns", Studies in Conservation, 34, p. 189-200.

Wouters J. et Verhecken A., 1989-2, "The Scale Insect Dyes (Homoptera: Coccoidea). Species Recognition by HPLC Diode Array Analysis of the Dyestuffs", Annales de la Société entomologique de France (N.S.), 25, p. 393-410.

Wouters J., 2001, “The Dye of Rubia peregrina. I. Preliminary investigations", Dyes in History and Archaeology, 16/17, p. 145-157.

Wouters J., Grzywacz C. M. et Claro A., 2011, "A Comparative Investigation of Hydrolysis Methods to Analyze Natural Organic Dyes by HPLC-PDA: Nine Methods, Twelve Biological Sources, Ten Dye Classes, Dyed Yarns, Pigments and Paints", Studies in Conservation, 56, p. 231-249.

\section{Documents inédits}

Ferreira E. S. B., 2002, New Approaches towards the Identification of Yellow Dyes in Ancient Textiles, The University of Edinburgh, Ph.D. Thesis.

Peggie D. A., 2006, The Development and Application of Analytical Methods for the Identification of Dyes on Historical Textiles, The University of Edinburgh, Ph.D. Thesis.

Sanyova J., 2001, Contribution à l'étude de la structure et des propriétés des laques de garance, Université Libre de Bruxelles, Ph.D. Thesis.

Troalen L. G., 2013, Historical Dye analysis: Method Development and New Applications in Cultural Heritage, The University of Edinburgh, Ph.D. Thesis. 